\title{
EARLY ONSET MARFAN SYNDROME: ATYPICAL CLINICAL PRESENTATION OF TWO CASES
}

\author{
Ozyurt $\mathrm{A}^{1, *}$, Baykan $\mathrm{A}^{2}$, Argun $\mathrm{M}^{2}$, Pamukcu $\mathrm{O}^{2}$, Halis $\mathrm{H}^{3}$, Korkut $\mathrm{S}^{3}$, \\ Yuksel $Z^{4}$, Gunes $\mathrm{T}^{3}$, Narin $\mathrm{N}^{2}$
}

*Corresponding Author: Abdullah Ozyurt, M.D., Division of Pediatric Cardiology, Erciyes University Faculty of Medicine, Kayseri, Turkey, 38039. Tel: +903522076666, Ext. 25036. Fax: +903524375825.

E-mail: duruozyurt@yahoo.com.tr

\begin{abstract}
Early onset Marfan Syndrome (eoMFS) is a rare, severe form of Marfan Syndrome (MFS). The disease has a poor prognosis and most patients present with resistance to heart failure treatment during the newborn period. This report presents two cases of eoMFS with similar clinical features diagnosed in the newborn period and who died at an early age due to the complications related to the involvement of the cardiovascular system.
\end{abstract}

Keywords: Craniosynostosis; Early onset Marfan Syndrome (eoMFS); Heart failure; Neonatal Marfan Syndrome (MFS); Pediatric; Supraventricular tachycardia

\section{INTRODUCTION}

Marfan syndrome (MFS) is an autosomal dominant disorder characterized by elastic tissue involvement: typically skeletal, cardiovascular, pulmonary, skin and ocular malformations occur.The disease is caused by mutations in $F B N 1$, encoding fibrillin

\footnotetext{
${ }^{1}$ Department of Pediatric Cardiology, Mersin Women Health And Children Hospital, Mersin, Turkey

${ }^{2}$ Division of Pediatric Cardiology, Erciyes University Faculty of Medicine, Kayseri, Turkey

${ }^{3}$ Division of Neonatology, Erciyes University Faculty of Medicine, Kayseri, Turkey

${ }^{4}$ Department of Medical Genetics, Mersin Women Health and Children Hospital, Mersin, Turkey
}

1 protein which provides force bearing structural support in elastic and nonelastic connective tissue, located on the chromosome $15 \mathrm{q} 21.1$. The prevalence of the disease has been reported as $0.02-0.03 \%$ and approximately $25.0 \%$ of patients are sporadic due to de novo mutations. Early onset MFS (eoMFS) represents the most severe end of the spectrum of MFS, which is clinically distinguished from the other forms by its association with severe mitral and/or tricuspid valve insufficiency showing a relentless progression resulting in heart failure and death within the first 2 years of life [1]. Early onset MFS is usually caused by de novo mutations between exons 24 and 32 of FBN1 $[2,3]$. Early diagnosis in the neonatal period and the initiation of anticongestive treatment are crucial to prevent the early development of the refractory heart failure in the newborns. This study presents two cases of eoMFS and a review of the literature.

\section{CLINICAL REPORT}

Case 1. A 30-day-old female infant was born to a 21-year-old (gravida 3, parity 2) healthy mother through spontaneous vaginal delivery at week 39 of gestation. The prenatal history of the mother indicated a regular prenatal care and a fetal ultrasound which could not find any abnormalities. There was no consanguinity between the parents and both of them were phenotypically normal. The infant was admitted to the pediatric emergency service with palpitation on the 30th day after birth. On admission, her medical state was serious and she was pale in appearance. 

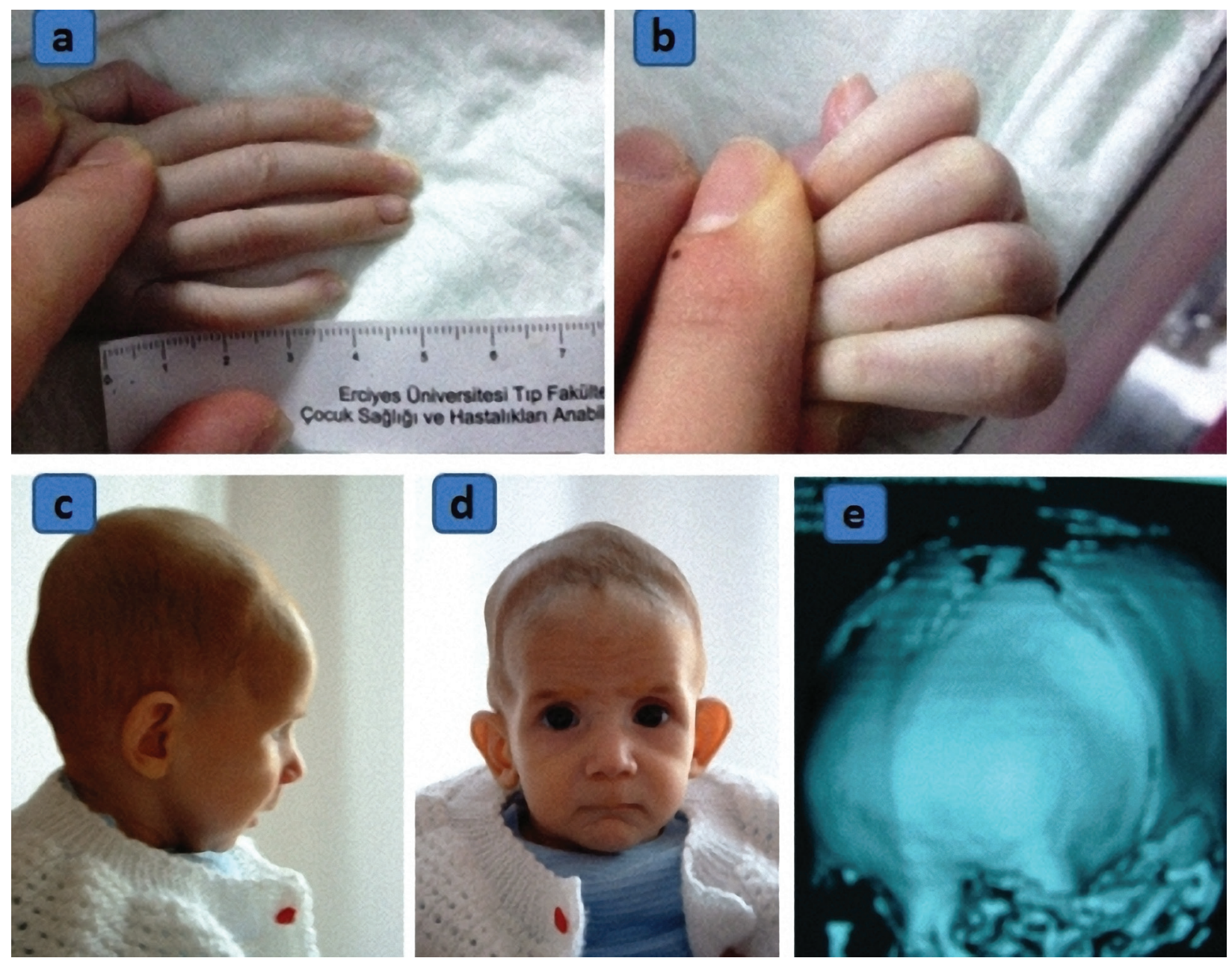

Figure 1. Clinical features of the patients: a) arachnodactyly of patient 1 ; b) Steinberg sign of patient 1; c-d) lateral and frontal views of the patient 2; e) craniosynostosis seen in 3D CT of patient 2.

The size of the anterior fontanelle was measured as $2 \mathrm{~cm}$ (mean) and the size of the posterior fontanel was measured as $0.5 \mathrm{~cm}$ (55th centile). On physical examination, arachnodactyly in fingers and toes (Figure $1 \mathrm{a}$ and $1 \mathrm{~b}$ ), prominent forehead, flat nose, high palate, low-set ears, enophtalmos, dolicocephaly, articular laxity particularly in wrists and ankles and a wrist sign was noted. Her weight was $4060 \mathrm{~g}$ (7597th percentile), height was $62 \mathrm{~cm}$ ( $>97$ th percentile), arm span was $65 \mathrm{~cm}$ ( $>97$ th percentile) (arm span/ height ratio: 1.04), upper segment (US) was $40 \mathrm{~cm}$, lower segment (LS) was $25 \mathrm{~cm}$ (US/LS: 1.6). No pathological reflex was detected in her neurological examination, however, a generalized hypotonia was noted. Heart auscultation revealed a 3/6 systolic murmur along the left sternal border and a gallop rhythm. The liver was palpable approximately $4 \mathrm{~cm}$ below the right costal margin. The heart rate was 168 / min., respiratory rate was found as $70 / \mathrm{min}$. The chest
X-radiograph (CXR) revealed cardiomegaly. She underwent an echocardiographic evaluation that demonstrated dextrocardia, mitral valve prolapses (MVP) (Figure 2a and 2b), severe mitral insufficiency (Mi) (Figure 2c), moderate tricuspid insufficiency, slightly insufficient aortic valve (Ai) (Figure 2c) and dilated left ventricle (LVEDd: $23 \mathrm{~cm}, \mathrm{Z}$ score: 3.1 ); aortic root measurements were as follows: Ao: $12 \mathrm{~mm}, \mathrm{Z}$ score: 2.17 . The patient was diagnosed with eoMFS based on the clinical and echocardiographic findings. The patient's blood level of homocysteine was found within normal limits. The eye examination did not reveal any abnormality. A mega cisterna magna was detected by transfontanelle ultrasound examination. She developed a supraventricular tachycardia (SVT) on the second day of observation. She was diagnosed with nosocomial pneumonia on the 20th day after the admission. She developed respiratory arrest on the 25th day and consequently she was intubated 


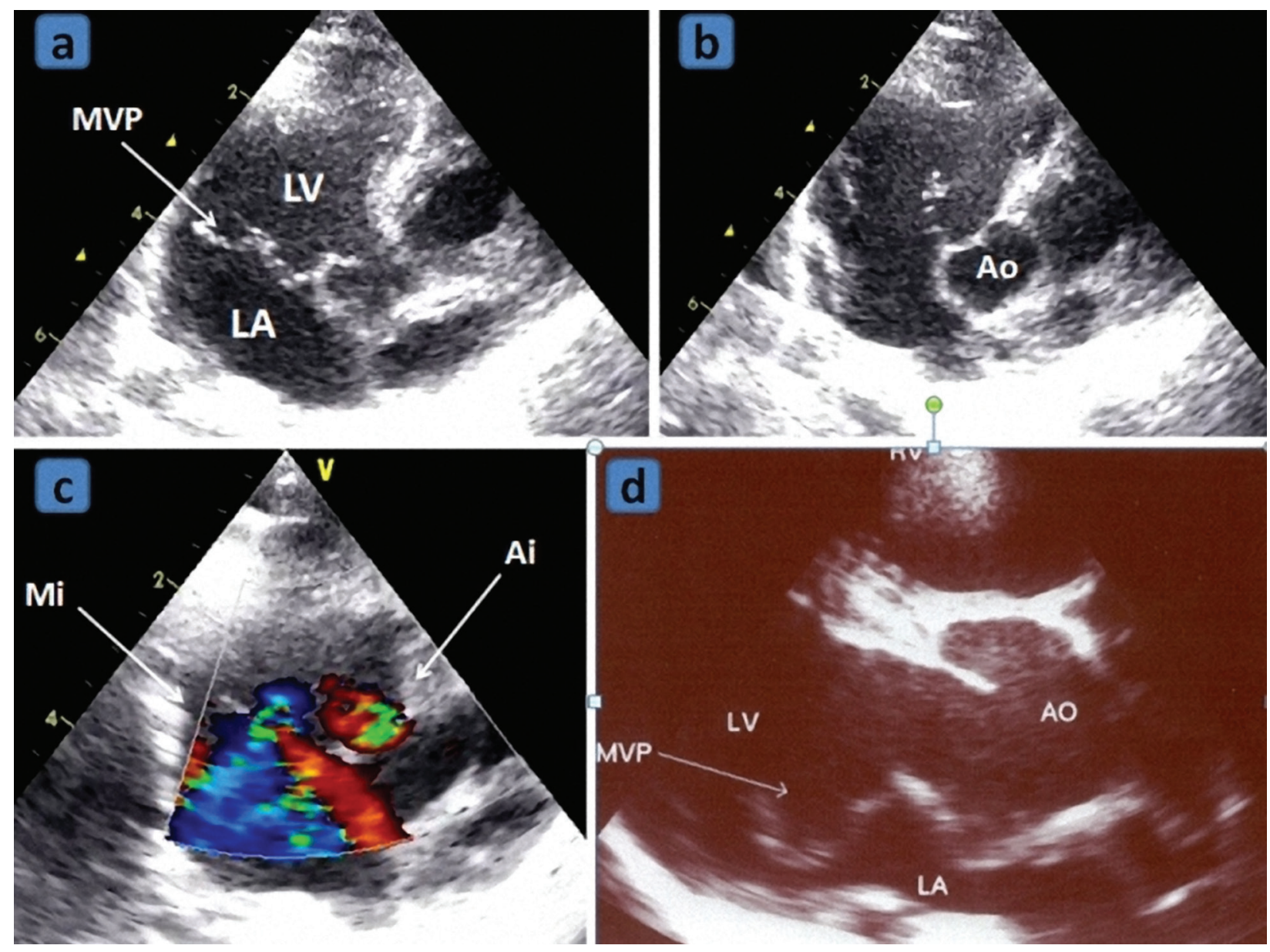

Figure 2. Echocardiographic features of patients: a, b, c) apical 5 chamber view of patient 1. Mitral valve prolapsus was seen in 2a and $\mathbf{2 b}$; aortic and mitral insufficiency seen in color Doppler echocardiography examination in $2 \mathrm{c}$. d) apical 5 chamber view of patient 2 .

and mechanical ventilator support was provided. The infant died on the 38th day after her admission (68 days after birth) due to multiple organ failure resulting from cardiogenic shock.

Case 2. A 4-month-old boy was admitted with history of nausea, vomiting, cough and lack of appetite. Marfanoid features were detected on physical examination. His facial features were typical of MFS, the leg and arm length were measured as 27 and $20 \mathrm{~cm}$, respectively. The US/LS was 0.74 (normal: $<0.85$ ), arachnodactyly and positive thumb signs were present, the joints were hyperextensible. The subcutaneous fat tissue was decreased. He had dolichocephaly with large frontal fontanel $(1 \times 2$ $\mathrm{cm}$ ) along with craniosynostosis (Figure 1c, 1d and 1e). Chest examination revealed a pectus excavatum deformity. In his cardiac examination, a 3/6 systolic murmur was heard on the left sternal border. The CXR revealed right pericardial infiltration, cardio- megaly, aortic root dilatation and left atrial enlargement. An echocardiogram demonstrated a normal sinus rhythm with a right axis deviation, biatrial dilatation and biventricular hypertrophy. Aortic root dilatation, MVP, minimal aortic valve insufficiency, severe mitral valve insufficiency and patent foramen ovale were detected in echocardiographic evaluation. Cranial computerized tomography (CT) scans revealed craniosynostosis in metopic and bilateral coronal sutures. Due to his Marfanoid features along with MVP and aortic root dilation, the patient was diagnosed as eoMFS.

\section{DISCUSSION}

In the present study, we report the clinical characteristics of two patients diagnosed as eoMFS according to the typical phenotypic appearance and radiological evaluation. The "classical" MFS is a 
rare (an incidence of 1-3/10,000), autosomal dominant disorder caused by mutations in the $F B N 1$ gene located on chromosome $15 \mathrm{q} 21.1$ that encodes the protein fibrillin-1. Pathogenesis is related to abnormal biosynthesis of fibrillin-1, which is the major constituent of elastin, the main structure in the elastic tissues. Therefore, musculoskeletal, central nervous and cardiovascular systems are in the first place; additionally, eyes, lungs, skin and other systems can be affected by the disease [3-5].

The term eoMFS has been used instead of neonatal MFS that was more commonly used previously. The eoMFS is a rare form of the classical MFS and differentiates from the latter in terms of genetic, phenotypic and prognostic features [4]. Joint contractures, mitral valve prolapse, and mitral, tricuspid, and pulmonary regurgitations were more prevalent in eoMFS. However, aortic regurgitation is less frequent. The prognosis in eoMFS is much worse than that of the classical MFS, the average age of death has been reported as 16.3 months in eoMFS, while classic MFS patients may live several decades [6]. Although both are autosomal dominant, approximately $75.0 \%$ of classical MFS cases are inherited, whereas eoMFS is predominantly sporadic. While only two cases of familial eoMFS were reported in the literature, one of these cases was reported from Turkey [7,8]. Although mutations have been observed along the entire length of the fibrillin-1 gene (FBN1) in classical MFS, mutations in eoMFS cluster in a relatively small region of $F B N 1$, usually between exons 24 and 32 [1,3-5]. The present study suggests that enhanced proteolytic susceptibility, especially in the linker region between TB3 and cbEGF11, and functional loss between central fibrillin-1 and heparin/heparan sulfate interactions contribute to the development of the more severe eoMFS as compared to MFS [3].

Consistent with the medical literature, congestive heart failure secondary to the atrioventricular valve involvement and severe atrioventricular (AV) valve insufficiencies, was the main feature in both cases. There was no family history in both cases. Since the genetic studies are not covered by health insurance in our country and the families could not afford the expenses, genetic studies could not be performed.

The diagnosis of MFS relies on typical dysmorphic features and diagnostic studies such as echocardiography. Genetic studies are used to support the diagnosis and to provide prenatal genetic counseling to the family in future pregnancies. The dysmorphic features-based Ghent criteria have been used in the diagnosis of MFS and were revised in 2010 [9]. According to the Ghent criteria, the positivity of two out of four criteria consisting of an aortic diameter at the sinuses of Valsalva above the indicated Z score or aortic root dissection, ectopialentis, systemic features $(>7)$ and positive FBN1 mutation, is considered as sufficient for the diagnosis of classical MFS in patients without a family history (Table 1). According to Loeys et al. [10], eoMFS is not considered as a separate category, but rather represents the most se-

Table 1. Scoring of systemic features.

\begin{tabular}{|l|c|}
\hline Dysmorphic Features & Scores \\
\hline Wrist and thumb sign (1 point each) & 3 \\
\hline Pectus carinatum deformity [pectus excavatum or chest asymmetry (1 point)] & 2 \\
\hline Hind foot deformity [plain pes planus (1 point)] & 2 \\
\hline Pneumothorax & 2 \\
\hline Dural ectasia & 2 \\
\hline Protrusioacetabuli & 2 \\
\hline Reduced US/LS and increased arm/height and no severe scoliosis & 1 \\
\hline Scoliosis or thoracolumbar kyphosis & 1 \\
\hline Reduced elbow extension & 1 \\
\hline Facial features (3/5) (dolichocephaly, enophthalmos, down slanting palpebral fissures, malar hypo-plasia, retrognathia) & 1 \\
\hline Skin striae & 1 \\
\hline Myopia $>3$ diopters & 1 \\
\hline Mitral valve prolapse (all types) & 1 \\
\hline
\end{tabular}

US: upper segment; LS: lower segment. 
vere end of the MFS spectrum. As the current studies have indicated, the Ghent criteria should be revised for the neonatal form of the disease.

The clinical features depend on the degree of the affected organ systems. Marfan syndrome is an autosomal dominant disorder characterized by elastic tissue disorder, but various mutations may cause clinically different phenotypes, and consequently, MFS has a wide clinical spectrum. The association of SVT and MFS was documented in two cases in the medical literature and one of these patients was successfully treated by catheter ablation $[10,11]$. In another study, the presence of a variety of arrhythmias was shown in $13 \%$ of the patient with MFS [12]. The impaired conduction system in MFS may be secondary to the severe atrial and ventricular dilation resulting from severe valve insufficiencies or primary involvement of the conduction system characterized as fibrous tissue. On the other hand, craniosynostosis and dolichocephalyare not common in MFS, and these dysmorphic skeletal features have been reported in a few cases in the literature [13]. These findings can be suggested as a rare finding of eoMFS.

Several conditions have been recognized that present overlapping clinical manifestations with MFS in the cardiovascular, ocular or skeletal systems. These include conditions associated with aortic aneurysms [Loeys-Dietz syndrome (LDS), bicuspid aortic valve, familial thoracic aortic aneurysm, valvu- lar Ehler-Danlos syndrome (vEDS), arterial tortuosity syndrome], ectopialentis (ectopialentis syndrome, Weill-Marchesani syndrome, homocystinuria, Stickler syndrome) or systemic manifestations of MFS [Shprintzen-Goldberg syndrome, congenital contractural arachnodactyly, LDS, MASS phenotype and Mitral Valve Prolapsus syndrome (MVPS)] (Table 2).

The eoMFS has variable clinical presentations and should be taken into consideration in the differential diagnosis of connective tissue disorders. Supraventricular tachycardia and craniosynostosisdolichocephaly may be associated with eoMFS. Certainly, current approaches in the treatment and diagnosis of this severe disorder will be developed by sharing the experiences of large prospective case series including successful management of the disease.

Declaration of Interest. The authors report no conflicts of interest. The authors alone are responsible for the content and writing of this article.

\section{REFERENCES}

1. HennekamRC. Severe infantile Marfan syndrome versus neonatal Marfan syndrome. Am J Med Genet A. 2005; 139(1): 1.

2. Tiecke F, Katzke S, Booms P, Robinson PN, Neumann L, Godfrey M, et al. Classic, atypically

Table 2. Features of differential diagnosis with early onset Marfan syndrome.

\begin{tabular}{|c|c|c|}
\hline Differential Diagnosis & Gene & The Main Distinctive Features \\
\hline Loeys-Dietz syndrome (LDS) & $T G F B R 1 / 2$ & $\begin{array}{l}\text { bifid uvula/cleft palate, arterial tortuosity, hyper-telorism, } \\
\text { diffuse aortic/ and arterial aneurysms, craniosynostosis, } \\
\text { clubfoot, cervical spine insta-bility, thin and velvety skin, } \\
\text { easy bruising }\end{array}$ \\
\hline Shprintzen-Goldberg syndrome (SGS) & FBN1 and others & craniosynostosis, mental retardation \\
\hline Congenintal contractural arachnodactyly (CCA) & $F B N 2$ & crumpled ears, contractures \\
\hline Weill-Marchesani syndrome (WMS) & FBN1, ADAMTS10 & microspherophakia, brachydactyly, joint stiffness \\
\hline Ectopialentis syndrome (ELS) & $F B N 1, L T B P 2$ & lack of aortic root dilatation \\
\hline Homocystinuria & $A D A M T S L 4, C B S$ & thrombosis, mental retardation \\
\hline Familial thoracic aortic aneurysm syndrome (FTAA) & TGFBR1/2, ACTA2 & $\begin{array}{l}\text { lack of Marfanoid skeletal features, levidoreticu-laris, } \\
\text { irisflocculi }\end{array}$ \\
\hline FTAA with bicupid aortic valve (BAV) & - & - \\
\hline FTAA with patent ductus arteriosus (PDA) & MYH11 & - \\
\hline Arterial tortuosity syndrome (ATS) & $S L C 2 A 10$ & $\begin{array}{l}\text { generalized arterial tortuosity, arterialstenosis, facial } \\
\text { dysmorphism }\end{array}$ \\
\hline $\begin{array}{l}\text { Ehlers-Danlos syndrome } \\
\text { (vascular, valvular, kyphoscoliotictype) }\end{array}$ & $\begin{array}{l}\text { COL3A1, COL1A2, } \\
\text { PLOD1 }\end{array}$ & $\begin{array}{l}\text { middle sized artery aneurysm, severe valvular insuffiency, } \\
\text { translucent skin, dystrophic scars, facial characteristics }\end{array}$ \\
\hline
\end{tabular}


severe and neonatal Marfan syndrome: Twelve mutations and genotype phenotype correlations in FBN1 exons 24-40. Eur J Hum Genet. 2001; 9(1): 13-21.

3. Kirschner R, Hubmacher D, Iyengar G, Kaur J, Fagotto-Kaufmann C, BrömmeD, et al. Classical and neonatal Marfan syndrome mutations in fibrillin-1 cause differential protease susceptibilities and protein function. J Biol Chem. 2011; 286(37): 32810-32823.

4. Apitz C, Mackensen-Haen S, Girisch M, Kerst G, Wiegand G, Stuhrmann M, et al. Neonatal Marfan syndrome: Unusually large deletion of exons 24-26 of FBN1 associated with poor prognosis. Klin Pediatr. 2010; 222(4): 261-263.

5. Faivre L, Masurel-Paulet A, Collod-Beroud G, Callewaert BL, Child AH, Stheneur C, et al. Clinical and molecular study of 320 children with Marfan syndrome and related type I fibrillinopathies in a series of 1009 probands with pathogenic FBN1 mutations. Pediatrics. 2009; 123(1): 391-398.

6. Milewicz DM, Duvic M. Severe neonatal Marfan syndrome resulting from a de novo 3-bp insertion into the fibrillin gene on chromosome 15 . Am J Hum Genet. 1994; 54(3): 447-453.

7. Strigl S, Quagebeur JM, Gersony WM. Quadrivalvar replacement in infantile Marfan syndrome. Pediatr Cardiol. 2007; 28(5): 403-405.
8. Tekin M, Cengiz FB, Ayberkin E, Kendirli T, Fitoz S, Tutar E, et al. Familial neonatal Marfan syndrome due to parental mosaicism of a missense mutation in the FBN1 gene. Am J Med Genet A. 2007; 143A(8): 875-880.

9. Elshershari $H$, Harris C. Paternal fibrillin-1 mutation transmitted to an affected son with neonatal marfan syndrome: The importance of early recognition. Cardiol Young. 2014; 24(4): 735-738.

10. Loeys BL, Dietz HC, BravermanAC, Callewaert BL, De Backer J, Devereux RB, et al. The revised Ghent nosology for the Marfan syndrome. J Med Genet. 2010; 47(7): 476-485.

11. Frank RE Jr. Supraventricular tachycardia vs. Marfan's syndrome. J Insur Med. 1997; 29(3): 204-207.

12. Yetman AT, Temple J, Erickson CC. Radio frequency ablation of a left-sided atrioventricular pathway in a patient with Marfan syndrome. Cardiol Young. 2002; 12(5):494-495.

13. Porciani MC, Attanasio M, Lepri V, Lapini I, Demarchi G, Padeletti L, et al. Prevalence of cardiovascular manifestations in Marfan syndrome. Ital Heart J Suppl. 2004; 5(8): 647-652.

14. Mégarbané A, Hokayem N. Craniosynostosis and marfanoid habitus without mental retardation: Report of a third case. Am J Med Genet. 1998; 77(2): 170-171. 